\title{
Die Entwicklung der Physiologie im Spanien des 19. Jahrhunderts
}

\author{
Von José L. Barona Vilar
}

Die Einführung der experimentellen Physiologie in der zweiten Hälfte des 19. Jahrhunderts leitete einen Wechsel der Perspektiven in der Beobachtung und in der Erklärung biologischer Prozesse ein. Zweifellos ist eine der ausschlaggebendsten Ursachen dieses Ereignisses in der allmählichen Einführung von Arbeitsmethoden zu sehen, die in enger Beziehung zur naturwissenschaftlichen Denkweise schließlich den physiologischen Determinismus konzipierten und ihn als theoretische Grundlage einer Forschung einführten, die sich auf analytische Experimente stützte ${ }^{1}$. Gleichzeitig brachte dies einen grundsätzlichen Orientierungswechsel mit sich; bisher war die Wissenschaft hauptsächlich damit beschäftigt, die Bedingtheiten und Einflüsse der physikalischen Welt gegenüber den Lebewesen abzugrenzen. Deren Funktionen glaubte man von Gesetzen determiniert, die wesensverschieden waren von jenen, die die leblose Materie beherrschten. Der sogenannte physiologische Reduktionismus war die Bemühung um die Erklärung sämtlicher energetischen und materiellen Umwandlungen, welche die Lebensvorgänge charakterisieren. Dies wäre nicht möglich gewesen ohne das Antreten einer allgemeinen Physiologie, die ihre Fragen aus einer weitschichtigeren Perspektive stellt, und die dem Tierexperiment einen Sinn zuschreibt.

Diese neue Orientierung, die den Kurs der Humanphysiologie in Richtung der experimentellen Disziplinen leitete, forderte zweifelsohne die systematische Eingliederung der konzeptuellen und technischen Mittel, die dank der progressiven Entwicklung der Naturwissenschaften dem Studium der Lebensfunktionen angeglichen werden konnten. Darin liegt begründet, daß nur eine soziale Mentalität, die diesen theoretischen Voraussetzungen günstig gesonnen war und sich in der Lage sah, den durch die Laborforschung geforderten und gleichzeitig notwendigen Institutionalisierungsprozeß zu unterstützen, die soziale Integration fördern konnte und somit den Grundstein einer soliden Professionalisierung legte. Die enorme Entwicklung, die während der zweiten Hälfte des 19.Jahrhunderts durch die analytische Forschung in der Physiologie, die der Kenntnis über jede einzelne der organischen Funktionen neue Wege öffnete, erreicht worden war, darf nicht losgelöst betrachtet werden vom Institutionalisierungspro- 
zeß, der dank der Disziplin in den größten Teilen Europas durch Experimente erreicht worden war ${ }^{2}$. Das Deutschland um die Jahrhundertmitte stellt an sich das kennzeichnendste Beispiel dar: Eine beispielhafte institutionelle Planung, die zur Schaffung von Institutionen, autonomen Lehrstühlen und Laboratorien an den wichtigsten Universitäten führt, begleitet von einer herausragenden Kreativität im Erfinden von neuen Experimentierinstrumenten. Dies ermöglichte ein wirkliches Aufblühen der Kenntnisse über die Physiologie des Menschen. Die vorliegende Abhandlung will die Frage beantworten, inwieweit die spanische Gesellschaft des 19. Jahrhunderts in der Lage war, die adäquaten Voraussetzungen für diese Umwandlung auf dem Gebiete der physiologischen Forschung während des vergangenen Jahrhunderts zu schaffen.

\section{Ende der Aufklärungsphysiologie und Periode entscheidender Krisis}

\section{Das Modell institutioneller Organisation}

Die soziale Unbeständigkeit, die hartnäckige ideologische Unterdrückung und die unzulängliche institutionelle Organisation erschwerten die Entwicklung der physiologischen Forschung in Spanien. Obwohl sich schon einige Studienpläne um die Jahrhundertwende (18. bis 19. Jahrhundert), so z. B. der Plan des Direktor Blasco für die Universität von Valencia zur Aufgabe setzten, Amphitheater zwecks praktischer Vivisektion zu errichten, war doch die Lehre der Physiologie durch die ausschließlich theoretische Unterweisung stark eingeengt ${ }^{3}$.

Zu Beginn des 19. Jahrhunderts war einer der wesentlichen Störfaktoren das Nebeneinanderbestehen zweier Berufe: der des «reinen» Arztes und der des Chirurgen, die über eigene und separate Lehrinstitutionen verfügten. Diese Unterscheidung begünstigte zweifellos Rivalitäten und beeinträchtigte die Möglichkeit einer entscheidenden Universitätsreform, die auf der Basis einer beruflichen Vereinheitlichung eine Besserung der Studienbedingungen vorsah.

Die Spannungen führten während den ersten Jahrzehnten zu einer unaufhörlichen Reihe von Auflösungen und Restaurationen, die doch nur das treue Spiegelbild eines anachronistischen sozialen Systems waren, das unfähig war, einen soliden institutionellen Rahmen zu schaffen. Während die medizinischen Fakultäten über eine eher traditionelle Struktur verfüg- 
ten, zeigten die Escuelas de Cirugía (chirurgische Schulen), die im 18. Jahrhundert durch bourbonische Initiative gegründet worden waren, bessere Bedingungen für Assistenz und Lehre, nämlich dort, wo die unmittelbare Nachbarschaft eines Hospitals ermöglichte, dem praktischen Bereich des Lehramtes größere Aufmerksamkeit zu schenken ${ }^{4}$. Die folgenden Versuche einer Vereinheitlichung der Lehre scheiterten einerseits infolge der eigenen internen Spannungen und andererseits auf Grund des wirtschaftlichen Notstandes und des politischen Absolutismus, der im ersten Drittel des Jahrhunderts regierte. Unter diesen Umständen befand sich die Lehre der Physiologie in Händen derer, die sich des öftern ihrer Stellung als Mittelweg zu besser ausgestatteten Lehrstühlen bedienten, während wiederum andere nicht besetzt waren.

Es war daher nicht selten zu sehen, daß man der Forschung den Rücken kehrte und das Lehramt einem Zersetzungsprozeß ausgeliefert war, der dazu beitrug, daß sich dieses zu einem routinierten und memoristischen Verfahren entwickelte, in dem sehr oft die Prüfungsnoten von der obligatorischen Präsenz in den Klassen oder dem vom Professor geforderten Kauf seiner Texte abhingen.

Charakteristisch war auch die Schaffung medizinischer Akademien, deren Betrieb sich unabhängig von der Universität abspielte. In Spanien erschienen zuerst die Regia Sociedad de Medicina y demás Ciencias de Sevilla (1697), und später gründete man Akademien mit königlicher Unterstützung in den wichtigsten Städten Spaniens ${ }^{5}$. Bis Ende des 18. Jahrhunderts hatten sich die medizinischen Akademien durch ihre modernen Formen ausgezeichnet, im Zusammenhang mit einer dezentralisierten Organisation und am Rande der politischen Macht. Dennoch fanden die soziopolitischen Ereignisse, die eine tiefe Krisis der spanischen Naturwissenschaften während der ersten Jahrzehnte des 19. Jahrhunderts verursacht hatten, auch einen negativen Nachklang in den medizinischen Akademien, die nach der von Calomarde durchgeführten Reorganisation nun direkt der zentralen Macht unterstellt wurden.

Während des Década Absolutista (absolutistischen Jahrzehnts) (18231833) verwandelten sich die medizinischen Akademien in ausdrückliche Instrumente der ideologischen Repression, und nach dem Tode Fernando VII wurden sie zu rein symbolischen Institutionen herabgesetzt.

Schon zu Beginn des 19. Jahrhunderts war das wissenschaftliche Leben der Akademien, als dieses noch nicht vollständig unterbrochen worden war, in eine ausgesprochene Krise geraten. Die schwerwiegende soziale Unbestän- 
digkeit beraubte sie der notwendigen Unterstützung, und allmählich hörten ihre Sitzungen und die Veröffentlichung ihrer Annalen auf Grund fehlender wirtschaftlicher Einnahmen auf. Die Überlebensversuche stießen außerdem auf eine doppelte, religiöse und fakultative Zensur; die letztere zu Lasten einzelner, durch die politische Autorität designierter Ärzte.

Ab 1831 fand eine Reform statt, deren Folge die Eingliederung der Akademien in die entsprechenden provinzialischen Universitätsbezirke war. Gleichzeitig verwandelten sie sich - trotz ihrer theoretischen Befugnisse - in rein konsultative Organe, insbesondere in Themen der öffentlichen Gesundheit, deren Mittelpunkt oft ausschließlich rhetorische Diskussionen bildeten, weit entfernt von der sozialen und wissenschaftlichen Realität Europas.

\section{Die Veröffentlichung physiologischer Schriften}

Analysieren wir die Veröffentlichungen physiologischer Arbeiten im Sinne eines zusätzlichen Indikators der sozialen Eingliederung dieser Disziplin, so zeigt sich, daß die Daten den wissenschaftlichen Zusammenbruch der spanischen Gesellschaft zwischen 1801 und 1843 eindeutig bestätigen ${ }^{6}$.

Tabelle 1: In Spanien veröffentlichte Physiologie-Bücher (1801-1843), gegliedert nach historischen Perioden

\begin{tabular}{lll}
\hline Perioden & Originale & Übersetzungen \\
\hline Ende der Aufklärung (1801-1808) & 5 & 7 \\
Spanischer Unabhängigkeitskrieg (1809-1814) & 0 & 0 \\
Unter der Herrschaft Fernando VII: & & \\
- Rückkehr zum Ancien Régime (1815-1820) & 3 & 0 \\
- Dreijährige liberale Regierung (1821-1823) & 3 & 0 \\
- Das Absolutistische Jahrzehnt (1824-1833) & 3 & 3 \\
Die Regentschaften (1834-1843) & 2 & 7 \\
\hline
\end{tabular}

Fügt man diesen Daten den Niedergang des medizinischen Journalismus der Aufklärung hinzu, der sich bis 1840 nicht erholte, so läßt sich folgern, daß dië spanische Gesellschaft eine Periode wissenschaftlicher Isolierung durchlebte, die eine tiefgreifende Krise nach sich zog ${ }^{7}$. Die Übersetzungen waren nur spärlich vorhanden, zwischen 1800 und 1833 haben wir nur zehn 
lokalisieren können, von denen sieben vor 1808 bereits übersetzt worden waren. Es handelte sich hauptsächlich um für das Lehramt bestimmte Auszüge, die in den meisten Fällen aus französischen Autoren stammten. Unter den in der Universität verwendeten Textbüchern fanden sich Werke von Nicolas Adelon, Charles L.Dumas, François Magendie, Balthasar Richerand und Xavier Bichat, die alle zwischen 1803 und 1828 übersetzt worden waren.

Die Assimilation physiologischer Ideen dieser Autoren führte zur Veröffentlichung einiger, ebenfalls für das Lehramt bestimmter physiologischer Auszüge durch spanische Autoren. Unter ihnen können wir folgende zitieren: Elementa phisiologicae humanae (1819) von Félix Janer, Studienrat der Disziplin in Cervera, die hauptsächlich auf dem Animismus von Ernst Stahl basieren; die Elementos sucintos de fisiología (1822) von Juan Ribot y Mas; der Compendio de Fisiología (1817) von Juan Vicente Carrasco, eine Adaptation der Lehren von Ch. Dumas; der Compendio de Fisiología (1834) von Juan Coll y Feliu und die Phisiologia chimica del cuerpo humano (1804) von J.Ponce de León. Auch Eugenio de la Peña erarbeitete einige Lecciones de fisiología (nicht veröffentlicht), die er während seines Lehramtes im Colegio de San Carlos während der ersten Jahre des Jahrhunderts geschrieben hatte. Doch zweifelsohne verdienen, dank der damalig aktuellen Erneuerungsentwürfe ihrer Autoren, auch solche Werke erwähnt zu werden, die an der Universität gebraucht wurden, wie die Elementos de fisiología especial o humana (1830) von Juan Mosácula, Nachfolger Eugenios de la Peña im Lehrstuhl des Colegio de San Carlos. Mosácula erkannte als erster die Notwendigkeit, das Studium der spanischen Physiologie auf der Grundlage der Laborforschung zu erneuern. Dennoch wurde sein Versuch der Umgestaltung des Lehramtes der Physiologie schnell entmutigt, da er sowohl verfolgt als auch seines Postens während der ideologischen Repression unter Fernando VII enthoben worden war. Nach seiner Rehabilitation starb er sehr frühzeitig mit $36 \mathrm{Jahren}$. Die tragischen sozialen Bedingtheiten, die die Pläne Mosáculas mißlingen ließen, unterdrückten gleichzeitig seit Beginn des Jahrhunderts auf dem Gebiete der Physiologie jegliche Möglichkeit der Entwicklung einer experimentellen Arbeitsrichtung in Spanien ${ }^{8}$.

Der Nachfolger Mosáculas im Lehrstuhl der Physiologie am Colegio de San Carlos war Joaquín Hysern, der den Versuch, die Physiologie dem Labor zu nähern, wieder aufnahm, nachdem er bei François Magendie in Paris gearbeitet hatte. Sein Einsatz stieß jedoch auf den Mißmut und die 
Verständnislosigkeit seiner Arbeitskollegen, wie man eindeutig den Vorwürfen gegen ihn entnehmen kann:

\begin{abstract}
«Doktor Hysern wäre ein durchaus bemerkenswerter Professor seiner Disziplin, würde er sich ausschließlich einem Lehrstuhl seines Faches widmen; um nun aber die Elemente der Physiologie zu erklären, um Menschen zu schaffen, die später fähig sein sollen, den komplizierten Pfad des Rationalismus zu beschreiten, ist er zu fahrlässig, schenkt er allzu viel Bedeutung den Experimenten, und die Zeit, die man in der Vorbereitung derselben verschwendet, ist im Abhalten von Vorlesungen, so wie es die Beschaffenheit seines Lehrstuhls verlangt, nicht nachzuholen.» ${ }^{9}$
\end{abstract}

Die Denkart, die aus diesem Auszug spricht, ist ein Zeichen dafür, daß während der Jahrhundertmitte in Spanien und insbesondere unter den Ärzten, eine der Scholastik nahestehende und bereits antiquierte Auffassung des Lehramtes und der Ausübung der Physiologie gepflegt wurden. Zweifellos erschwerte die positive Bewertung der «Doktrin» gegenüber der Ablehnung der Forschung im Laboratorium die Konsolidierung der experimentellen Forschung, wie sie in andern Ländern praktisch vollzogen wurde.

\title{
Die physiologischen Kenntnisse
}

Während der ersten Hälfte des 19. Jahrhunderts in Spanien blieben die allgemeinen Entwürfe, die die Physiologie der Aufklärung erarbeitet hatte, und die hauptsächlich über den Weg der französischen Physiologie assimiliert worden waren, auch weiterhin in Kraft. Ihr theoretisches Fundament ging von einer vitalistischen Auffassung der organischen Funktionen aus, die die Existenz eines qualitativen Unterschieds zwischen den Lebewesen und der unbelebten Materie postulierte: Die Lebenserscheinungen fordern entweder mechanische oder chemische Wechselvorgänge heraus, doch handelt es sich hauptsächlich um vitale Handlungsabläufe, deren Erklärung fordert, daß man sich an die Existenz einer Eigenheit oder wesentlichen Kraft der Stoffe hält, die auf die eigentümliche Anordnung der lebendigen Materie zurückzuführen ist.

Von diesem Standpunkt ausgehend, setzte man mit dem Studium jeder einzelnen organischen Funktion ein, die in zwei großen Absätzen jeweils behandelt werden: die Funktionen der Nahrungsstoffe und die Funktionen des Beziehungslebens. Die nicht geradewegs unkomplizierte Erklärung der notwendigen Verbindung zwischen beiden fand ihre Lösung darin, daß man jedem einzelnen Teil des Organismus eine spezifisch vitale Eigenschaft zurechnete, die ihnen gleichzeitig die adäquate Sensibilität verlieh, um auf 
die spezifischen Reize dieser Zone zu antworten, und so ihrer Funktion gemäß zu reagieren. Dieses fundamentale Schema der internen organischen Funktionen beinhaltete zwei Aspekte: einerseits ermöglichte die experimentelle Forschung zu erfassen, was dieses Phänomen veranlaßte, andererseits mußte man den letzten und orientierenden Ursprung dieser Erscheinung in der Vitalität der Gewebe suchen.

Im Falle Mosáculas ist der Blickpunkt seines Werkes im historischen Kontext seiner Epoche als eindeutig modern zu bezeichnen: beeinflußt durch den Orientierungswechsel, den die französische Physiologie erfuhr, betrachtete er die Humanphysiologie schon als tragendes Fundament der Pathologie - eine Idee, die einige Jahrzehnte später ausgearbeitet wurde und der sogenannten experimentellen Pathologie ihren Ursprung gab - und er unterstreicht die Notwendigkeit, das Konzept der Funktion zum Studium der organischen Störungen einzuführen.

Der Compendio de Fisiología (1817) von Juan Vicente Carrasco ${ }^{10}$ wurde seinerseits in der Mosáculas Werk direkt vorangegangenen Zeitetappe als Textbuch gehandhabt. Sein Autor, eng verbunden mit den Reales Hospitales de la Corte und der Real Academia Médica Matritense, erarbeitete eine Synthese der physiologischen Kenntnisse, basierend auf dem Werk von Charles Louis Dumas, Principes de physiologie ou l'introduction à la science expérimentale (Paris, 1800-1803). Dumas Werk fand einen merklichen Nachklang in der Physiologie seiner Zeit. Womöglich besteht sein wichtigster Beitrag in der theoretischen Bearbeitung der Funktionen der «Lebenskraft» in den Eigenschaften der lebenden Gewebe: Die Reaktion auf Reize, die Assimilation oder die Resistenz.

\section{2. Überwindung der Isolierung: Die Funktion des medizinischen Journalismus}

Die 1843 erfolgte Modifikation der medizinischen Lehre (Plan Mata), beinhaltete eine Erneuerung sowohl der Studienpläne, als auch der eigenen Organisation der Universität. Ihre Anwendung versuchte sich in der Konsolidierung der professionellen Einigung von Ärzten und Chirurgen, in der Eingliederung der naturwissenschaftlichen Fächer in die medizinischen Studien, dehnte die Länge des Studiums aus und sah eine materielle Ausbesserung des Lehrstoffs voraus. Im Laufe des folgenden Jahrzehnts fand eine Vermehrung ${ }^{11}$ des Lehrkörpers statt, und zum ersten Mal erschien 
die Figur des Assistenten im experimentellen Unterricht der Physiologie, der abwechslungsweise als Ergänzung der theoretischen Stunden stattfinden sollte. Laut eines königlichen Erlasses (Real Orden), der einige Jahre später erschien, mußte die öffentliche Prüfung zum physiologischen Assistenten zwei Aufgabenstellungen beinhalten. Die erste, die allen Assistenzstellen gemeinsam war, bestand in einer theoretischen Prüfung, in der auf zehn von insgesamt zwanzig durch ein Losverfahren ausgewählte Fragen geantwortet werden mußte. Die zweite dagegen bestand in einer praktischen Arbeit, d.h. in der Durchführung einer von insgesamt drei ausgewählten Vivisektionen und der Beschreibung und Handhabung des Mikroskops in seiner fachlichen Anwendung ${ }^{12}$.

Nach den sechziger Jahren, und trotz der veralteten Textbücher, dem Mangel an Erklärungen und der experimentellen Notdürftigkeit, die das generelle Panorama aller Zentren bildete, hatte sich das Fach leicht erholt und gebessert, vor allen Dingen hatte man nun die Konsolidierung der beruflichen Einigung erreicht.

Um die Jahrhundertmitte wurden weiterhin solche Textbücher verwendet, wie die Auszüge von Juan Mosácula, Juan Vicente Carrasco und Juan Coll y Feliu ${ }^{13}$, außerdem einige ausländische Werke, hauptsächlich französische, die um diese Zeit übersetzt wurden. Unter den an der Universität meistbearbeiteten Autoren befinden sich: J.L. Brachet und A.D. Fouilhoux, Jules Béclard, Johannes Müller und Ludimar Hermann.

Dessen ungeachtet waren die von spanischen Autoren abgefaßten Auszüge nicht sehr zahlreich und ohne Originalität. Auf ein begrenztes Echo stießen die Lecciones de Fisiología (1848) von Juan Ribor y Ferrer ${ }^{14}$, die hauptsächlich für die Mittelschulen, bestimmt waren; der Ensayo de antropología o historia fisiológica del hombre (1844) von Juan Valera de Montes ${ }^{15}$; die Lecciones de física médica (1845) von José Gardoqui ${ }^{16}$, die den Unterrichtsstoff an der Universität von Cádiz zusammenfaßten und die Rudimentos de fisiología (1857) von Marcos Bertrán y Pastor ${ }^{17}$, die den bereits genannten Lektionen des Lehrstuhls der Physiologie von Barcelona entsprachen. Zweifellos war das meistverbreitete Werk der Tratado elemental de fisiología humana (1869) von Juan Magaz, der vier neubearbeitete Auflagen erreichte und als offizielles Textbuch erklärt wurde ${ }^{18}$. Das Werk vermittelt eine breite Information von der Physiologie dieser Epoche, obwohl es jeglicher Originalität entbehrt und den Prototyp des rein theoretischen Unterrichts an den spanischen Universitäten darstellt. Mit Magaz, Professor der Physiologie in Barcelona und später im Colegio de San Carlos, hatte sich 
der Bruch mit den sich abwechselnden Erneuerungsversuchen, die zuerst von Juan Mosácula und später, in den vorangehenden Jahrzehnten von Joaquín Hysern verteidigt worden waren, gefestigt. Bezeichnenderweise war er sowohl im Kreise der Ärzte, als auch in der spanischen Gesellschaft seiner Zeit hoch geschätzt.

Die medizinischen Akademien änderten ihre Organisation, und unter den neu geschaffenen Sektionen erschien eine, die der Anatomie und Physiologie gewidmet war. Dennoch hatten die Sitzungen, abgesehen von ihren Interventionen für die öffentliche Hygiene, auch weiterhin einen fast ausschließlich theoretischen Charakter, und die Auswahl ihrer Mitglieder hing davon ab, ob sie Anhänger der an der Universität praktizierten Medizin waren. Auf dem Gebiete der Physiologie war ihr Einfluß nur sehr spärlich, und fast immer standen sie am Rande des Fortschritts großer theoretischer Auseinandersetzungen und empirischer Erfindungen ${ }^{19}$.

Der medizinische Journalismus. Seit den zwanziger Jahren und insbesondere seit der Jahrhundertmitte fand ein bemerkenswerter Informationszuwachs dank den medizinischen Publikationen über Themen der Physiologie statt, obwohl noch keine spezialisierten Zeitschriften auf dem Markt waren. Die systematische Analyse von sechsundzwanzig medizinischen Zeitschriften, die in sieben Städten veröffentlicht worden waren, hat folgende Ergebnisse aufgezeigt:

Tabelle 2: Geschichtlich periodische Aufteilung im spanischen Fachjournalismus des 19. Jahrhunderts veröffentlichter Arbeiten über Physiologie

\begin{tabular}{lcccc}
\hline Perioden & Originale & $\begin{array}{l}\text { Über- } \\
\text { setzungen }\end{array}$ & $\begin{array}{l}\text { Nach- } \\
\text { richten }\end{array}$ & $\begin{array}{l}\text { Buch- } \\
\text { anzeigen }\end{array}$ \\
\hline Dreijährige liberale Regierung (1821-1823) & \multicolumn{1}{c}{1} & 3 & 3 & 1 \\
Das Absolutistische Jahrzehnt (1824-1833) & 0 & 3 & 9 & 0 \\
Die Regentschaften (1834-1843) & 2 & 7 & 19 & 3 \\
Die Regierung Isabel II (1844-1868) & 75 & 8 & 227 & 30 \\
Sechsjährige Revolutionsperiode (1869-1874) & 20 & 4 & 88 & 5 \\
Die Restauration der Monarchie (1875-1900) & 139 & 38 & 746 & 82 \\
\hline
\end{tabular}

Die Anfänge unter der Regierung Isabel II (1844-1868) bedeuteten eine regelrechte Revolution auf dem Gebiete der Information, und es fanden Diskussionsrunden über neu gewonnene physiologische Kenntnisse statt. 
Dennoch hinderte eine fehlende institutionelle Reform die Einführung experimenteller Arbeiten in Spanien, und noch Ende der sechziger Jahre sprach man von einem informationsfreudigen Land, ohne selber Bücher über physiologische Forschung zu schreiben.

Neben den Arbeiten von Juan Magaz - das für die Universitätslehre bestimmte Werk enthält bereits einige Elemente der Zelltheorie, die aus der Sicht des Vitalismus auf die Physiologie angewandt werden -, waren um diese Zeit auf dem akademischen Gebiet auch Schriften von José Varela de Montes und Marcos Bertrán y Pastor verbreitet. Der Ensayo de Antropología o Historia Fisiológica del Hombre (1843) von Varela de Montes, Oberstudienrat von Santiago, überschreitet die Grenzen einer rein physiologischen Abhandlung, indem er versucht, eine globale Vision des Menschen insbesondere aus einer physiologischen Sicht - in Bezug zu seiner Umgebung, zum Universum und zur Krankheit zu bieten. Dies alles leitete er ab aus einem vitalen, d.h. nicht materiellen Lebensprinzip und stets im Einvernehmen mit der christlichen Sicht des Menschen. Seinerseits verfaßte Bertrán auf nur sehr begrenzter empirischer Basis und mit einer starken Dosis Philosophie, die didaktisch ausgezeichneten Rudimentos de Fisiología (1857). Die spanische Physiologie blieb der Theorie enger verbunden als den Experimenten.

\section{Institutionelle Reform und Experimente im Laboratorium}

1868 fand in Spanien eine revolutionäre Umwälzung statt, die 1873 durch die Ausrufung der 1. Republik gekrönt wurde. Diese Bewegung sozialer Erneuerung traf mit dem fortschrittlichen Gedankengut eines Ärzteteams zusammen, das sich der Dekadenz der gültigen institutionellen Ordnung widersetzte und in Verbindung mit den europäischen experimentellen Zentren stand. Dies ermöglichte, dank der Einführung der Lehrfreiheit, die Schaffung von Institutionen nichtoffiziellen Charakters, die der Lehre und der Forschung gewidmet waren. Dazu gehören das Instituto Biológico de Madrid, Escuela Libre de Medicina de Sevilla, Escuela Libre de Medicina del Museo Antropológico de Madrid. Diese zwischen 1868 und 1875 neu geschaffenen Institutionen sahen eine radikale Modernisierung des Lehramtes vor und waren sich einig, die experimentelle Beweisführung der Lehre der Physiologie einzugliedern, indem nach dem Modell von Carl Ludwig in Leipzig neue Forschungslaboratorien geschaffen wurden. 
Die Escuela Libre de Medicina de Sevilla ${ }^{20}$ wurde 1868 durch die Initiative von Federico Rubio geschaffen ${ }^{21}$. Sie war mit einem Arbeitszimmer für die Physiologie, einem Laboratorium, einem Amphitheater zur Demonstration der Vivisektionen und einem für die Experimente bestimmten Tiergarten ausgestattet. Für das Lehramt der Physiologie war José Moreno Fernández ${ }^{22}$ verantwortlich, Verfasser des ersten spanischen Kompendiums zur generellen Physiologie im J. 1879, Lecciones de fisiología general (1879), und Verteidiger der analytischen Experimente, die aus der Sicht des Positivismus eine revolutionäre Bewegung auf dem Gebiet der Physiologie nach sich gezogen hatten ${ }^{23}$.

Als Initiant des Instituto Biológico de Madrid fungierte Rafael Martínez Molina und mit ihme eine ausgewählte Gruppe von Vertretern der medizinischen und biologischen Experimente ${ }^{24}$. Unter den von spanischen Autoren während des 19. Jahrhunderts veröffentlichten Werken, stellt das Tratado elemental de fisiología general von Balbino Quesada ${ }^{25}$ eine der herausragendsten Abhandlungen über generelle Physiologie dar. Sein Werk zeugt von einer gründlichen Kenntnis und vollkommenen Assimilation der Ideen von Claude Bernard und ist sich der physiologischen Konsequenzen einer darwinistischen Evolutionstheorie voll bewußt ${ }^{26}$. Die Arbeit von Moreno Fernández und Quesada und die geschichtliche Bedeutung der Institutionen, in denen sie arbeiteten, zogen sowohl die Einführung der generellen Physiologie in das wissenschaftliche Kulturleben Spaniens, als auch eine wichtige Unterstützung zu Anfang der experimentellen Arbeit im Labor nach sich. Dies geschah einerseits im Einklang mit der Orientierung der Escuela Práctica Libre del Museo Antropológico de Madrid, die von Pedro González de Velasco ${ }^{27}$ geschaffen worden war, und andererseits in scharfem Widerspruch zur Haltung der Universitätsphysiologie, die sich auf das reine Bücherstudium berief.

Bei den angewandten Texten handelt es sich normalerweise um Übersetzungen oder Adaptationen ausländischer, fast immer französischer Werke, und man hatte noch nicht die Einrichtung physiologischer Laboratorien erreicht, die die notwendige technische Ausstattung aufweisen konnten. Die Vorlesungen der Professoren, die während des letzten Jahrhunderts Lehrstühle der Physiologie an spanischen Universitäten besetzten, bezeugen diese Neuerungen und genügen, um ihre mangelhafte Originalität zu bestätigen, nicht nur in Bezug auf die Forschung, sondern auch im Assimilationsprozeß der experimentellen Physiologie. Der Ausgangspunkt der experimentellen Physiologie an der spanischen Universität ist im Werk von 
José Gómez Ocaña zu sehen, der dank seiner persönlichen Initiative eine originale Forschungsarbeit um die Jahrhundertwende begann und gleichzeitig Kontakte zu den wichtigsten europäischen Figuren der Physiologie seiner Zeit knüpfte ${ }^{28}$. Gómez Ocaña erscheint als erster spanischer Repräsentant auf den internationalen Kongressen für Physiologie, an deren Organisationskomitees er bei mehreren Gelegenheiten beteiligt war, und seine Fisiología humana teórica y experimental (1896) bedeutete schon eine Aktualisierung der Kenntnisse in dieser Materie. Außerdem trug Gómez Ocaña, Oberstudienrat der Physiologie zuerst in Cádiz, später in Madrid, zum Studium der optischen Lokalisation des Gehirns bei, zur Erklärung der Funktion des Nervus Vagus und führte weiterhin eine Serie von Arbeiten über die Drüsen innerer Sekretion aus, speziell der Schilddrüse, die später auf eine große Nachfolgerschaft in der physiologischen Tradition Spaniens stie $\beta^{29}$.

\section{Auftreten der Humanphysiologie und ihre Institutionalisierung im Spanien des 19. Jahrhunderts}

Die soziale Unbeständigkeit, die schwere politische Unterdrückung und die unzulängliche institutionelle Organisation waren von bedeutender Tragweite für die physiologische Forschung. Der Zusammenbruch der wissenschaftlichen Aktivität Spaniens unter der Regierung von Fernando VII, bedeutete für die spanische Physiologie einen schweren Schlag, von dem sie sich nur allmählich erholte. Die Lehre der Physiologie an den medizinischen Fakultäten und chirurgischen Schulen erreichte nicht, die Enge exklusiv theoretisch geführter Vorlesungen zu überwinden, die an direkt von der zentralen Macht abhängigen Institutionen erteilt wurden, die erschöpft waren durch den wirtschaftlichen Ruin und bestimmt durch den Streit zwischen den sogenannten «reinen Ärzten» und den Chirurgen.

Erst nach der endgültigen Vereinigung beider Berufe ab 1843 erreichte man eine gewisse Stabilität, die man hätte ausnützen können, um die Fundamente eines Orientierungswechsels der offiziellen Physiologie zu legen. Dennoch behielten auch weiterhin die Zensurmechanismen und die Auflegung von Textbüchern ihre Gültigkeit, und zusammen mit der wirtschaftlichen Krise erschwerten sie die Möglichkeiten eines Modernisierungsprozesses und aller Anreize zur Forschung. Erst Ende des Jahrhunderts, und mit merklicher Verspätung in bezug auf andere Gebiete der Medizin und der 
Naturwissenschaften, konnte die Universitätsphysiologie über die notwendigen technischen Mittel und das adäquate Personal verfügen, um eine originelle Forschungsarbeit zu beginnen. Die allmähliche Einführung der neuen analytischen Physiologie auf experimenteller Basis in Spanien erforderte das Zusammentreffen von zwei Faktoren: eine Gruppe erneuerungsfreudiger und der gültigen institutionellen Ordnung entgegengesetzter Ärzte, und der entsprechende soziale Rahmen, der erst ab 1868 nach der Modernisierung der spanischen Gesellschaft entstand.

Diese Situation ermöglichte die Schaffung neuer Institutionen, die losgelöst von der zentralen Macht und dank herausragender Persönlichkeiten, die sich der Notwendigkeit des Wechsels bewußt waren, sowohl über die notwendigen technischen Mittel, als auch über die notwendige Freiheit verfügten, um den Erneuerungsprozeß zu beginnen. Diese Arbeit wurde auf dem Gebiet der Physiologie nicht durch große Leute in Gang gesetzt, sondern befand sich generell in Händen wenig bekannter Männer, die sich in der Lage fühlten, eine Disziplin zu lehren, die der offiziellen Unterstützung entbehrt. Nur während der Restauration der Monarchie ermöglichte der Rahmen sozialer Stabilität bessere Austauschmöglichkeiten mit dem Ausland und folglich eine Annäherung zur Forschung im Laboratorium. Dank der institutionellen Reformen fanden wesentliche Wechsel in den experimentellen Methoden statt und der informative Wiedergewinnungsprozeß, der unter der Regentschaft Isabell II begonnen worden war, setzte die adäquaten Austauschmöglichkeiten mit dem Ausland fest. Die Generation der Physiologen der Jahrhundertwende (José Gómez Ocaña, Balbino Quesada, José Moreno Fernández ...) fanden Arbeitsansätze wieder, die mit dem Tode von Juan Mosácula aufgegeben worden waren. 


\section{Anmerkungen}

1 In diesem Sinne wird auf die wichtige theoretische Arbeit von Claude Bernard verwiesen, als einem der fundamentalen Initianten dieser Erneuerung.

2 Dieser Aspekt wurde eingehend von Karl Rothschuh bearbeitet in seinem Werk: Rothschuh, K., Geschichte der Physiologie, Berlin 1953; insbesondere die Kapitel VI und VII.

3 Zum eingehenderen Studium der medizinischen Lehre in Spaniens 19. Jahrhundert lese man: Comenge, L., La medicina en el siglo XIX. Apuntes para la historia de la cultura médica en España, Barcelona 1914, Espasa; Peset, M. und Peset, J. L., La enseñanza de la medicina en España durante el siglo XIX. Med.Esp. 60 (1968) 28-35; 98-105; Peset, J. L., La enseñanza de la medicina en España durante el siglo XIX. Minoría de Isabel II. Regencia y gobierno provisional (1833-1843). Med.Esp. 63 (1970) 115-130; Peset, M., und Peset, J.L., La Universidad española (siglos XVIII-XIX.) Despotismo Ilustrado y revolución liberal. Madrid 1974, Taurus.

Auf dem Gebiet der Physiologie: Barona, J.L., El cultivo de la fisiología en las instituciones españolas del siglo XIX. Asclepio 37 (1985) 183-208.

4 Unter den verschiedenen Arbeiten, die den spanischen Reales Colegios de Cirugía gewidmet sind, erwähnen wir: Aparicio Simón, J., Historia del Real Colegio de San Carlos de Madrid, Madrid 1956; Ferrer, D., Historia del Real Colegio de Cirugía de la Armada de Cádiz, Barcelona 1961; López Rodríguez, A., El Real Colegio de Cirugía de Cádiz y su época. Sevilla 1969; Usandizaga Soraluce, M., Historia del Real Colegio de Cirugía de San Carlos de Madrid (1787-1828). Madrid 1984; Usandizaga Soraluce, M., Historia del Real Colegio de Cirugía de Barcelona (1760-1843). Barcelona 1964.

5 Bujosa, F., La Academia Médico-Práctica de Mallorca (1788-1800). Valencia (1975), und Comenge, L., (1914) op. cit., bieten eine Gesamtübersicht zum Thema des königlichen Betriebs der medizinischen Akademien.

6 Eine eingehendere Analyse über die Veröffentlichung physiologischer Arbeiten in Spaniens 19. Jahrhundert, in: Barona, J. L., La fisiología humana en la sociedad española del siglo XIX. Valencia 1983, Tesis doctoral.

7 Die auf anderen Gebieten der spanischen Wissenschaft des 19. Jahrunderts realisierten Arbeiten zeigen, daß es sich in Wirklichkeit um eine bemerkenswerte Krise der wissenschaftlichen Aktivität handelt, als Konsequenz einer sozialen, wirtschaftlichen und im weiteren Sinne politischen Krise.

8 Eine ausführliche Analyse über das Werk Mosáculas in: Barona, J. L., La obra fisiológica de Juan Mosácula Cabrera (1794-1831), Llull 7 5-27.

9 El Crisol, 1 (1855) 7.

10 Carrasco, Juan Vicente, Compendio de fisiología o conocimiento del hombre físico y vital. 2 Bände, Madrid 1817, Imprenta de José Callado.

11 Die gültige Reglementierung für die Vergebung neuer Lehrstühle erscheint, in: Reglamento para la provisión de Cátedras de las Universidades, Escuelas Superiores y profesionales e Institutos de segunda enseñanza, y para las traslaciones, ascensos y jubilaciones de los catedráticos, Madrid 1864, Of. Tip. del Hospicio.

12 Cf. Barona, J. L. (1985), op. cit., S. 183-190. 
13 Coll y Feliu, Juan, Compendio elemental de fisiología. Barcelona 1834, Imprenta de A. Berger.

14 Ribot y Ferrer, Juan de Dios, Lecciones de fisiología. 3. Auflage, Barcelona 1848, Imprenta J.M. de Grau.

15 Varela de Montes, José, Ensayo de Antropología, o sea historia fisiológica del hombre en sus relaciones con las ciencias sociales y especialmente con la patología y la higiene. 4 Bände, Madrid 1844, Bailly-Baillière.

16 Gardoqui, José de, Lecciones de física médica dadas en la Facultad de Cádiz. Cádiz 1845, Imprenta y Librería de la Revista Médica.

17 Bertrán y Pastor, Marcos, Rudimentos de fisiología. Barcelona 1857, Imprenta Politécnica de Tomás Gorch.

18 Über Leben und Werk des Juan Magaz, $c f$. Barona, J.L., Magaz y Jaime, Juan. In: Diccionario Histórico de la Ciencia Moderna en España. 2 Band, S. 13-15. Barcelona 1982, Editorial Península; und Barona, J.L., El «Tratado elemental de fisiología humana» de Juan Magaz y la fisiología académica española durante la segunda miytad de siglo XIX. Med. Esp. (im Druck).

19 Cf. Barona, J. L. (1985), op. cit., S. 191-193.

20 Bis zum heutigen Moment fehlt eine umfassende Arbeit über die Institution von Sevilla; dennoch war ihr Eingriff auf dem physiologischen Gebiet das Objekt einer eingehenden Analyse in: Barona, J. L., La Escuela Libre de Medicina de Sevilla, y la introducción de la mentalidad experimental en la fisiología española del siglo XIX. San Sebastian (1984), Akten zum III Congreso de la Sociedad Española de Historia de las Ciencias.

21 In bezug auf Federico Rubio, $c f$. López Piñero, J.M.: Rubio Galí, Federico. In: Diccionario Histórico de la Ciencia Moderna en España. 2. Band, S.269-272; Sánchez de la Cuesta, G., Ideario y grandeza de Don Federico Rubio. Sevilla, Real Academia de Medicina.

22 In bezug auf José Moreno Fernández, cf. Barona, J.L.: Moreno Fernández, José. In: Diccionario Histórico de la Ciencia Moderna en España. 2. Band, S. 85-86.

23 Moreno Fernández, José. Lecciones de fisiología general. Sevilla 1879, Imprenta de R.Tarascó.

24 Über Leben und Werk des Rafael Martínez Molina, $c f$. López Piñero, J.M.: Martínez Molina, Rafael. In: Diccionario Histórico de la Ciencia Moderna en España. 2. Band, S.36-38; Pulido Fernández, A., La Medicina y los Médicos, Valencia (1883), Lib. Pascual Aguilar, und auch Homenaje a la memoria del Doctor Martínez Molina, Madrid 1901, Asilo de Huérfanos del Sagrado Corazón.

25 Quesada Agius, Balbino, Tratado elemental de fisiología general. Madrid 1880, Establecimiento Tipográfico de Eduardo Cuesta.

26 Leben und Werk des Balbino Quesada sind Objekt einer eingehenden Studie in: Barona J.L., Vida y Materia en el «Tratado elemental de fisiología general» de Balbino Quesada. Dynamis 3 (1983) 172-198.

27 Über Leben und Werk von Pedro González de Velasco, sf. López Piñero, J.M.; González de Velasco, Pedro. In: Diccionario Histórico de la Ciencia Moderna en España. 1.Band, S.417-420; Pulido Fernández, A., El doctor Velasco. Madrid (1894), Imp. Enrique Teodoro.

$28 C f$. Ruiz de Galarreta, A., José Gómez Ocaña. Su vida y su obra. Arch., Iber. Hist. Med. y Antr. Med. 10, 379-496. 
29 Eine Studie über das Werk von Gómez Ocaña und seine Bedeutung für die Geschichte der spanischen Physiologie, in: Barona, J.L., La fisiología humana en la sociedad española del siglo XIX, und speziell das letzte Kapitel.

\section{Summary}

The social instability of the Spanish society during most of the 19th century (economical decline, ideological censorship, political absolutism) lead to an institutional crisis as well as to the collapse of the practice of experimental sciences. During the first part of the century, physiology was strongly influenced by this general situation. The absence of experimental work reduced Spanish physiology to a bookish science used at the teaching centres. Free institutions were founded after the 1868 Revolution. This lead to the beginning of a radical modernisation of physiological laboratories. The present paper analyses the introduction of modern physiology into 19th century Spain. There follows a study of the institutions, the exchange of information and the evolution of the theoretical background.

Professor Titular, Dr. med. José Luis Barona Vilar

Departamento de Historia de la Ciencia

(Avda. Blasco Ibañez, 17, 46010 Valencia)

Universidad de Valencia

Valencia, Spain 